\title{
El discurso docente en clases de lenguaje y matemáticas en un tercero básico chileno: un estudio de caso
}

Pérez-Sobarzo, Fernanda; Yancovic-Allen, Macarena

El discurso docente en clases de lenguaje y matemáticas en un tercero básico chileno: un estudio de caso Revista Educación, vol. 45, núm. 2, 2021

Universidad de Costa Rica, Costa Rica

Disponible en: https://www.redalyc.org/articulo.oa?id=44066178008

DOI: https://doi.org/10.15517/revedu.v45i1.42376

\section{(c) $(1) \Theta$}

Esta obra está bajo una Licencia Creative Commons Atribución-NoComercial-SinDerivar 3.0 Internacional. 


\title{
El discurso docente en clases de lenguaje y matemáticas en un tercero básico chileno:
} un estudio de caso

\author{
Teacher Discourse for Third Grade Language Arts and Mathematics Classes in the Chilean School System: A Case \\ Study
}

Fernanda Pérez-Sobarzo

Universidad Finis Terrae, Chile

fer.perezsobarzo@gmail.com

iD https://orcid.org/0000-0002-9580-4882

Macarena Yancovic-Allen

Universidad Finis Terrae, Chile

myancovic@uft.cl

https://orcid.org/0000-0002-3179-817X
DOI: https://doi.org/10.15517/revedu.v45i1.42376

Redalyc: https://www.redalyc.org/articulo.oa? $\mathrm{id}=44066178008$
Recepción: 26 Junio 2020

Aprobación: 01 Octubre 2020

\section{Resumen:}

Conocer las interacciones entre estudiantes y el profesorado es fundamental para el proceso de enseñanza-aprendizaje, puesto que la calidad de las interacciones dentro del aula definirá los procesos pedagógicos (Mourshed, Chijioke y Barber, 2012). A través de un estudio de caso único, se indaga de manera profunda en el discurso de una docente de tercero básico en dos unidades didácticas, una de Matemáticas y la otra de Lenguaje. Para esto, se estudia el tiempo e intencionalidad de su discurso y se analiza el tipo de preguntas que realiza, para así identificar las habilidades cognitivas que estas promueven en el alumnado. Los datos fueron recolectados a través de grabaciones de audio, transcritos y posteriormente analizados. Los resultados evidencian que la docente domina la clase tanto en el tiempo discursivo como en el tipo de preguntas que realiza, las que son mayoritariamente cerradas, centradas en el contenido y que promueven habilidades cognitivas de bajo orden. Se concluye que este ámbito debe ser abordado e intencionado por las personas docentes, por lo que se recomienda comprender su importancia y trabajar en diseños de estrategias que promuevan habilidades cognitivas de orden superior en sus aulas y, a la vez, que esta temática sea abordada en los programas de formación inicial docente.

Palabras Clave: Docente, Discurso, Proceso de aprendizaje, Educación básica.

\section{ABstract:}

Understanding student and teacher interactions is an essential part of the teaching-learning process, given that quality of these interactions in the classroom define pedagogical processes (Mourshed, Chijioke and Barber, 2012). A third-grade teacher discourse when teaching Mathematics and Language Arts was analyzed with regards to length and intent as well as the types of questions posed to identify cognitive skills elicited among students. The obtained data was transcribed and analyzed from audio recordings. Based on the results, teacher discourse dominated classroom time and questions which were predominantly close-ended, contentfocused and more focused on the promotion of low-order cognitive skills. It was concluded that this aspect must be purposefully addressed by teachers. We, therefore, recommend that teachers gain insight early on in their training about designing strategies to promote higher-order cognitive learning in the classroom.

KEYWORDS: Teacher, Discourse, Learning Process, Primary School.

\section{INTRODUCCIÓN}

A través del paso de los años, es posible observar la intencionalidad de promover un cambio de paradigma en el sistema educativo chileno, en donde se han intentado modificar los roles que históricamente han 
desempeñado los y las docentes, esperando que sean mediadores/as del conocimiento y busquen desarrollar habilidades en el alumnado a través de los intercambios verbales (Martinic, Vergara y Huepe, 2013).

No obstante, la literatura ha dado cuenta que utilizan su habla mayoritariamente para entregar y comunicar contenidos, promoviendo de esta manera un pensamiento mecánico más que para fomentar un pensamiento reflexivo que intencione el desarrollo cognitivo de los y las estudiantes en sus clases (Preiss, Larraín y Valenzuela, 2011). Debido a esto, conocer las interacciones que se generan entre estudiantes y profesorado es fundamental para el proceso de enseñanza-aprendizaje, puesto que la calidad de estas dentro del aula definirá los procesos pedagógicos (Mourshed, Chijioke y Barber, 2012).

La literatura menciona que el tipo de interacción y relación que se establece entre dos o más interlocutores incide en gran medida en sus procesos cognitivos y en sus disposiciones emocionales hacia la propia interacción y hacia los contenidos que se abordan en sus intercambios comunicativos (Cuadrado y Fernández, 2008). Algunas características de las interacciones producidas en el aula son que se elaboran, forman y desarrollan con la participación conjunta de docentes y estudiantes en la medida en que avanza la clase, en donde se establece un doble proceso de construcción a través del diálogo; tanto del estudiante en sí como del profesor junto al estudiante, dándose una negociación de los significados, saberes escolares y dinámica del aula reguladas por la participación social (Goldrine y Rojas, 2007).

Lo anterior hace que sea relevante indagar en el discurso de las y los docentes, para fortalecer de esta manera el proceso de interacción de aula. Es por esto que la presente investigación indaga en el discurso de una docente de tercero básico en las asignaturas de Lenguaje y Matemáticas. Específicamente, busca identificar el tiempo e intencionalidad de su discurso y analizar el tipo de preguntas que realiza, para así identificar el nivel cognitivo que estas promueven en sus estudiantes.

El artículo se compone de cuatro apartados, siendo el primero la introducción, en donde se define la variable y se realiza una revisión de literatura que devela cómo ésta ha sido investigada. El segundo corresponde a la metodología, la cual establece el tipo de investigación, define y caracteriza a la participante, se enuncia la forma de levantamiento de datos y la forma de análisis. El tercero muestra los resultados del estudio de manera objetiva y finalmente, el cuarto apartado de discusión y conclusiones, relaciona los principales resultados con la literatura revisada seguida por las conclusiones, limitaciones y proyecciones.

\section{Discurso DOCENTE}

Dentro de la interacción de aula, el discurso de la y el docente tiene un rol fundamental y ha sido objeto de estudios desde su naturaleza, en donde este puede ser autoritario o dialógico. Por una parte, el discurso autoritario se caracteriza por ser directivo, autoritario (Wells y Mejía-Arauz, 2005), con poco diálogo y conexión con las y los estudiantes, quienes tienen un rol pasivo y realizan intervenciones que ocupan poco tiempo del total de habla de la clase (Martinic, 2015; Martinic et al., 2013; Hardman, Abd-Kadir y Tibuhinda, 2012; Pontefrac y Hardman, 2005). Scott (1998) menciona que el discurso autoritario es utilizado principalmente para transmitir información. Lo anterior surge a raíz del amplio uso de una metodología basada en actividades que se concentran en el saber más que en el saber hacer. Ejemplos de lo anterior son el exceso de dictados, extensas exposiciones de contenidos descontextualizados, lo que influye directamente en la participación oral y reflexión por parte de las y los estudiantes (Prieto, 2005; Pontefract y Hardman 2005). Por otra parte, existe un discurso dialógico, el cual se enfoca en la extensión y complejidad de la interacción. Chin (2007) profundiza en este tipo de discurso, señala que, a través de este, la/el profesor alienta a las/los alumnos a exponer sus ideas, explorar y debatir puntos de vista y sugerencias tentativas basadas en preguntas abiertas o genuinas, espontáneas y expresadas en frases u oraciones completas.

Específicamente, Mercer (2001) destaca que el o la docente domina dos tercios del habla en el aula y las/los estudiantes solo un tercio. Lo anterior eleva la importancia de que la/el profesor promueva la participación de las/los estudiantes, les anime a exponer sus ideas, explorar, debatir puntos de vista, entregar 
sugerencias tentativas, realizar preguntas abiertas o genuinas, espontáneas y expresadas en frases u oraciones completas. De esto se puede obtener una información valiosa sobre los procesos cognitivos que realicen las y los estudiantes y poder así identificar sus comprensiones acerca de la realidad (Chin, 2006; Prieto, 2005). En las aulas chilenas, se evidencia que en las clases predomina un diálogo autoritario, transmisivo (Martinic y Vergara 2007) en donde Martinic (2013) señala que "en promedio el habla del profesor ocupa la mayor parte del tiempo de la clase" (p. 131).

Históricamente, el diálogo ha sido la herramienta cultural y psicológica que permite la transformación del pensamiento; es el instrumento de mediación básico que posibilita el proceso de construcción de significados entre estudiantes y profesor, ya que, debido a su doble funcionalidad comunicativa y representativa, se pueden modificar, redefinir y reconstruir los significados mediante la interacción con otros y, también, es mediante el cual es posible evidenciar los procesos de apropiación y construcción del conocimiento por parte de los estudiantes (Colomina, Mayordomo y Onrubia, 2001).

Específicamente en el contexto educativo, el diálogo se define como la acción recíproca a través de la cual se desarrollan los contenidos de enseñanza y procesos cognitivos entre profesor-alumno (Wells y Mejía-Arauz, 2005). Goldrine y Rojas (2007) profundizan en esta definición, señalan que, en su discurso, "el docente hace visible su comprensión sobre los contenidos curriculares y negocia con los estudiantes el acercamiento a los contenidos" (p.178). Es precisamente en este escenario en donde docentes, estudiantes y contenidos forman un triángulo interactivo y el rol de cada uno de ellos es determinante en el proceso de construcción del aprendizaje. En este se espera que los estudiantes elaboren significados a partir de los contenidos trabajados en clases, a través de la mediación activa de la persona docente (Colomina, Mayordomo y Onrubia, 2001).

La relación triádica entre profesor, alumno y contenido mencionada previamente, hace posible que surja el término de interactividad, el cual se define como la articulación de las actuaciones del profesor y alumnos en torno a una tarea o un contenido de aprendizaje y el tipo de interacción y relación que se establece entre los interlocutores, lo que incidirá en sus procesos cognitivos y en sus disposiciones emocionales hacia la propia interacción y hacia los contenidos que se abordan en sus intercambios comunicativos (Cuadrado y Fernández, 2008; Colomina, Mayordomo y Onrubia, 2001). Sin embargo, el discurso de la persona docente pierde eficacia si los estudiantes no desempeñan un rol activo en su aprendizaje, debido a que este no se adquiere mediante la recepción de información a un almacén de conocimientos, sino que es mediante la relación de nueva información, nuevas experiencias y formas de comprensión (Pontefract y Hardman, 2005). Es acá en donde el habla de los y las estudiantes adquiere relevancia, debido a que es a través de ésta en donde se evidencia la comprensión que van haciendo de los contenidos.

En esta actividad discursiva, ambos hacen acuerdos para construir un entendimiento compartido de los contenidos, procedimientos y tareas escolares (Evnitskaya y Berger, 2017; Goldrine y Rojas, 2007).

\section{Elementos del discurso docente}

La situación comunicativa construida entre profesores y estudiantes ha sido estudiada y analizada a partir de tres elementos, siendo el primero, el tiempo de habla, el cual mide en unidades de tiempo las intervenciones realizadas tanto por docentes como por estudiantes, obteniendo la duración y la frecuencia de cada una de ellas, número de veces que se realiza un acto específico; la proporción de tiempo que ocupa, el lugar de su realización y las acciones que la preceden o las que se desarrollan posteriormente (Martinic et al., 2013), obteniendo los tiempos reales invertidos para el logro de aprendizajes (Martinic y Vergara, 2007).

Un segundo elemento se relaciona con la intencionalidad del discurso de ambos actores. En este, Goldrine y Rojas (2007) identifican si los y las docentes están entregando instrucciones o haciendo seguimiento a una tarea, organizando a los estudiantes para una labor específica, retroalimentando alguna intervención, o simplemente abordando algún contenido. 
El tercer y último elemento se relaciona con las preguntas emitidas por los y las docentes, las que son un componente clave en el aula que sirven para promover la construcción del conocimiento en los y las estudiantes, permitiendo explorar e identificar el nivel de comprensión que tienen, sus ideas y razonamientos para así promover el proceso de aprendizaje a través de la interacción verbal (Ajaja y Eravwoke, 2012; Chin, 2006; Zuleta, 2005). A través de ellas se puede intencionar que los y las estudiantes justifiquen sus respuestas, se problematicen y contrasten diversas ideas, alejándose de tener solamente un carácter evaluativo, sino que siendo un eslabón clave en el proceso de construcción del aprendizaje (Chin, 2007). Específicamente, Ajaja y Ervwoke (2012) establecen que cerca de un 21\% de la clase corresponde a preguntas formuladas por los docentes.

Por una parte, las preguntas se han estudiado según su estructura, siendo abiertas o cerradas. Las preguntas abiertas son aquellas que se caracterizan por tener más de una posibilidad de respuesta, facilitando seguimientos orientados a reformular las respuestas de los estudiantes. Por otra parte, las preguntas cerradas se caracterizan por tener una respuesta única o ser dicotómicas, que no requieren más de una o dos palabras (Radovic y Preiss, 2010, Pontefrac y Hardman, 2005). Diversos estudios han evidenciado que las preguntas cerradas tienen una alta presencia en las aulas por sobre las abiertas, sobrepasando el 85\% (Eliasson, Go\#ran y Sørensen, 2017; Hardman et al. 2012).

Por otra parte, las preguntas se han estudiado en función de las habilidades cognitiva que promueven (Tornero, Ramaciotti, Trufello y Valenzuela, 2015; Ajaja y Eravwoke, 2012; Lee, Kinzie y Whittaker, 2012; Hus y Kordigel, 2011; Reinsvold y Cochrane, 2011; Oliveira, 2010), siendo preguntas que promueven una alta demanda cognitiva aquellas que corresponden a peticiones o afirmaciones que impliquen el desarrollo de un concepto, análisis, creación, realización de una actividad de indagación o de un procedimiento práctico asociado a este. Por su parte, las preguntas que promueven una baja demanda cognitiva son aquellas en donde se solicita una información previamente entregada, confirman lo afirmado por el profesor o se orientan a la entrega de una respuesta instruccional, centrándose en los niveles basales de la Taxonomía de Bloom revisada por Anderson y Krathwohl en el año 2001.

Chen y Liang (2017) confirman que existe un predominio por parte de los profesores con respecto a las preguntas que se formulan en la clase. Además, los autores establecen que gran parte de estas promueven habilidades cognitivas de bajo nivel, caracterizándose por ser literales y tener como objetivo principal evaluar los conocimientos, siendo lo más comúnmente solicitado el recordar información. Otros estudios concuerdan con lo establecido previamente, señalando que una alta cantidad de preguntas están orientadas a monitorear el recuerdo de conceptos más que al desarrollo de la comprensión de estos, existiendo un predominio de preguntas cerradas y de bajo desafío cognitivo, las que generarían una menor participación de los estudiantes en los procesos de construcción del conocimiento (Rusk, Sahlström y Pörn, 2017; Sedova, Sedlacek y Savaricek, 2016; Smart y Marshall, 2013; Ajaja y Eravwoke, 2012; Radovic y Preiss, 2010). Además, señalan que hay un predominio de formas de seguimiento de las respuestas por parte del profesor, que se basan en la repetición y evaluación de estas.

Otras investigaciones se han centrado en indagar en las preguntas abiertas (o preguntas auténticas), en donde se concluye que son las que precisamente favorecerían el desarrollo de habilidades de alta exigencia cognitiva, sin embargo, son escasas dentro una sala de clases (Willemsen, Gosen, van Braak, Koole y de Glooper, 2018; Godoy, 2015). No obstante, no siempre estas preguntas promueven altos niveles cognitivos. Por ejemplo, Tornero et al. (2015) evidencian que, si bien en preescolar se utilizan más preguntas abiertas que cerradas, de igual modo estas tienden a promover un bajo nivel cognitivo.

\section{Factores que influyen en el discurso docente}

Se han identificado múltiples factores que intervienen en el discurso docente y en la oportunidad de promoverlos a través de una interacción discursiva. Según Villalta, Martinic y Guzmán (2011) estos se 
relacionan con políticas públicas, con el tamaño del curso, el tipo de dependencia de los establecimientos educativos, al currículum, a los textos escolares, entre otros. A la vez, se relacionan con un problema de gestión de parte de las y los profesores, ya que carecen de estrategias de organización que les permitan realizar una secuencia interactiva, respetando los momentos de la clase (inicio, desarrollo y cierre).

Debido a lo anterior, se ha evidenciado que el habla docente ocupa la mayor parte del tiempo, en donde se exponen materias o explican procedimientos, destaca el tiempo clasificado como silencio, intercambios simples que responden a instrucciones realizadas por el profesor de baja complejidad cognitiva (Martinic, 2015; Martinic et al., 2013). Además, destaca la rígida estructura comunicativa basada en el saber hacer condicionado por la cultura institucional de orden y normas (Villalta y Palacios, 2014; Villalta y Martinic, 2011).

Otros estudios reflejan que el habla del/la docente también se encuentra influenciado por factores tales como la organización y distribución del espacio en el aula (Díez-Palomar, García, Molina y Rué, 2010), y el clima positivo (Godoy, Varas, Martínez, Treviño y Meyer, 2016), en donde se ha observado que carecen de métodos de trabajo más participativos que otorguen protagonismo y autonomía a sus estudiantes, para aumentar así sus oportunidades de lograr aprendizajes de calidad (Muhonen, Rasku-Puttonen, Pakarinen, Poikkeus y Lerkkanen, 2017; Godoy, Varas, Martínez, Treviño y Meyer 2016).

De lo anterior se destaca la importancia de las actitudes, verbalizaciones y movimientos del profesor, los que también serán claves para que las y los estudiantes puedan visualizar un patrón y puedan anticiparse a lo esperado por la/el profesor, ya que el vocabulario puede obstaculizar el aprendizaje y la comprensión por parte de las/los alumnos en las escuelas (Barwell, 2015). La gestión de aula y relaciones sociales entre maestros/as y estudiantes en el aula son fuertes predictores del comportamiento de las/los actores y de la comunicación que se genere (Villalta, Martinic, Assael y Aldunate, 2018), por lo que se debe considerar la utilización de estrategias para mantener la disciplina y la motivación constante (Mumbembe, Adewale, Peter, Adeniyi y Olufunmilayo, 2017).

Como ejemplo de lo anterior, Poulou (2015) evidencia que las actitudes docentes como la insatisfacción, ser amonestador y estricto, se correlacionan positivamente con las dificultades emocionales y de comportamiento de los y las estudiantes. El autor explica que es debido a esto que el liderazgo positivo de la persona docente favorecerá y promoverá la comprensión por parte del estudiante, entregando a su vez posibilidades de desarrollar habilidades sociales.

Finalmente, es importante destacar que los diálogos, la actitud y el comportamiento discursivo que utilice el o la docente va a estar influenciado por la consideración que tengan ellos sobre las experiencias personales, sus contextos sociales y culturales. Contextualizar la enseñanza a partir desde la cotidianidad de los y las estudiantes promoverá el desarrollo de interacciones de calidad y la construcción del conocimiento entre ambos agentes (Rey-Herrera y Candela, 2013).

\section{Metodología}

La presente investigación utiliza una metodología cuantitativa y se anida bajo un enfoque interpretativo, el cual, a través de un estudio de caso único busca analizar el habla de los y las docentes desde varias aristas, permitiendo de esta manera indagar el fenómeno de manera profunda (Sandoval, 1996).

Esta investigación ahonda en el habla de una docente, la cual fue definida con base en los siguientes criterios de selección: (a) Ser docente que trabajara en colegio municipal, (b) Ser docente que se desempeñara en tercer año básico, (c) Ser docente que impartiera las asignaturas de lenguaje y matemáticas, las que son evaluadas en Chile a través de una prueba estandarizada de carácter censal. De acuerdo con lo anterior, la participante de esta investigación es una docente de género femenino que cuenta con 24 años de experiencia en aula. Estudió Pedagogía en Educación Básica con especialización en Lenguaje en un Instituto Profesional y, a nivel laboral, trabaja desde hace 8 años en un colegio municipal de la zona sur de Santiago de Chile. Al momento 
de la investigación, la docente se desempeñaba como profesora-jefe de un tercero básico. Además de las asignaturas de Lenguaje y Matemáticas, impartía las asignaturas de Ciencias Naturales, Ciencias Sociales, Educación Tecnológica, Educación Física y Artes. La docente accedió a participar en esta investigación de manera voluntaria, firmando un consentimiento informado.

Se analizaron un total de dos unidades didácticas, conformadas por cuatro clases cada una. Una unidad pertenecía a la asignatura de Matemáticas y, la otra, a la asignatura de Lenguaje. Cada clase tuvo una duración promedio de 90 minutos, lo que en Chile corresponde a dos horas pedagógicas. Las clases fueron audiograbadas y posteriormente transcritas en su totalidad, procurando así plasmar la información de la manera más fidedigna. Para entregar confiabilidad a las clasificaciones realizadas, dos codificadores analizaron las transcripciones. En una primera instancia trabajaron de manera individual, para luego comparar y estimar la concordancia de las codificaciones. Este proceso que se llevó a cabo midiendo el Kappa de Cohen (Torres y Perera, 2009) obteniéndose un promedio de 0.77, lo cual es considerado como excelente (Fleiss, 1981). Cada análisis se realizó en coherencia con cada uno de los objetivos delimitados para esta investigación, los que hacen referencia a las siguientes temáticas:

Tiempo de habla: Para este objetivo se calculó el tiempo de habla en minutos y segundos de la clase en su totalidad y, de manera específica, se identificó el tiempo de habla de la persona docente, de las y los estudiantes y finalmente, aquel sin interacciones (Martinic y Vergara, 2007).

Intencionalidad del habla: Para este objetivo se utilizaron seis categorías, cinco de ellas basadas en Goldrine y Rojas (2007), las cuales son contenidos, tareas, procedimientos, seguimiento y retroalimentación y organización del trabajo. Se realizaron tres acciones que modificaron las categorías originales. En primer lugar y para ofrecer una mayor riqueza de la información, se optó por unir tareas y procedimientos, debido a que en ambas categorías hacen referencia a las labores propiamente de las actividades curriculares que se realizan durante la asignatura.

En segundo lugar, se separó seguimiento y retroalimentación, definiendo al primero como un mecanismo de control de la clase y al segundo, como aquella situación en donde la/el docente responde a las/los estudiantes en torno a una intervención de carácter académico, como por ejemplo, ampliando la información que uno de las/los estudiantes entregue. En tercer y último lugar, se integró enmarcamiento (Villalta y Martinic, 2011), el cual hace relación a aquellas intervenciones del/la docente de índole no académico o curricular.

Preguntas del docente: Para este objetivo, se analizaron las preguntas formuladas por este actor desde tres aristas: en primer lugar, se identifica el tipo de preguntas de acuerdo con su apertura (Pontefrac y Hardman, 2005); luego, la habilidad cognitiva que estas promueven en las/los estudiantes basado en la taxonomía de Anderson y Krathwohl (Tornero et al. 2015).

\section{Resultados}

La presente investigación indaga en el habla de una docente de tercero básico en la implementación de una unidad didáctica en las asignaturas de Matemáticas y Lenguaje.

Con respecto al tiempo de habla, se puede apreciar que el promedio de duración de las ocho clases analizadas corresponde a 89,5 minutos, dentro de los cuales el docente habla 38,4 minutos del total del tiempo con una diferencia de 1,6 minutos, predominando levemente en Matemáticas (37,6 minutos en Lenguaje y 39,2 minutos en Matemáticas). Los y las estudiantes tienen un tiempo de habla promedio de 13,2 minutos, mostrando una mayor participación en la asignatura de Lenguaje, superando en 10 minutos aproximadamente a la asignatura de Matemáticas (18,1 minutos en Lenguaje y 8,3 minutos en Matemáticas). Finalmente, el tiempo sin interacciones corresponde a 35,2 minutos, siendo este mayor en la asignatura de Matemáticas, con una variación de aproximadamente 4 minutos con la asignatura de Lenguaje (33,2 minutos en Lenguaje y 37,1 en Matemáticas). 
Con respecto a la intencionalidad del discurso docente, se evidencia que en ambas asignaturas predomina tareas y procedimientos, teniendo una mayor presencia en la asignatura de Lenguaje, superando a Matemáticas por un 3\%. La segunda categoría que predomina es la de Enmarcamiento, teniendo una presencia similar en ambas asignaturas (variación de 2,8\%). Con cifras más descendidas y con porcentajes similares se encuentra la Organización del trabajo (superando Matemáticas en un 1,6\% a Lenguaje) y el Seguimiento (promediando en ambas asignaturas un 12\%). Posteriormente, se encuentra el Contenido de la clase, en donde la asignatura de Matemáticas tiene una presencia que casi duplica a Lenguaje. Finalmente se encuentra la Retroalimentaciónen donde, si bien se observa que tiene una mayor presencia en la asignatura de Lenguaje, en ambas no superan el 10\%. La Tabla 1 entrega información específica sobre la intencionalidad del habla de la docente en ambas asignaturas.

TABLA 1.

Intencionalidad del habla docente.

\begin{tabular}{|c|c|c|c|}
\hline & Lenguaje \% & Matemáticas \% & Ejemplo \\
\hline Contenido & 6,1 & 11,4 & $\begin{array}{l}\text { L: la hipérbole es propia de la poesía (CL3, } \\
\text { L25) }\end{array}$ \\
\hline Tarea y procedimientos & 33,9 & 30,3 & $\begin{array}{l}\text { M: Lo voy a explicar ahora gráficamente en } \\
\text { la pizarra. (CM4, L102) }\end{array}$ \\
\hline Seguimiento & 12,2 & 12,1 & M: ¿Alguna duda? (CM2, L89) \\
\hline Retroalimentación & 9,7 & 4,5 & $\begin{array}{l}\text { M: Correcto (CM3, L54) ya lo hiciste } \\
\text { verdad. . hiciste esto y bien correcto y el } \\
\text { naranjo bien correcto, sigue. ¿De qué otra } \\
\text { manera lo puedes hacer? (CM1, L73) }\end{array}$ \\
\hline Organización & 13,1 & 14,0 & $\begin{array}{l}\text { L: Usted va a leer primero y después usted } \\
\text { (CL2, L11) }\end{array}$ \\
\hline Enmarcamiento & 25,0 & 27,8 & $\begin{array}{l}\text { L: Pensaba conseguir el parlante para } \\
\text { ensayar el baile (Fiesta nacional chilena) } \\
\text { (CL3, L121). }\end{array}$ \\
\hline
\end{tabular}

Fuente: Elaboración propia, basada en Goldrine y Rojas (2007)

En relación con las preguntas realizadas por la docente, en ambas unidades didácticas formuló un total de 478 preguntas, las que corresponden a un promedio de 11,2\% del tiempo total de las clases. En Lenguaje formuló 269 preguntas y, en Matemáticas, 209. Si bien existe una diferencia en el número de preguntas formuladas en ambas asignaturas, se aprecia un predominio de preguntas de carácter cerrado por sobre las de carácter abierto. Específicamente, en la asignatura de Matemáticas, un 76,1\% de las preguntas realizadas fueron de carácter cerrado y un $23,9 \%$ de carácter abierto. En el caso de la asignatura de Lenguaje, un 72,9\% de las preguntas formuladas por la docente fueron de carácter cerrado y un 27,1\% de carácter abierto.

Con respecto a las habilidades cognitivas que promueven las preguntas, se puede observar que, tanto en la asignatura de Lenguaje como en Matemáticas, existe un predominio de preguntas de baja demanda cognitiva ( $83 \%$ y $86 \%$ respectivamente). Específicamente, más del $65 \%$ de las preguntas que la docente realiza se focalizan en la habilidad de Recuperar, en donde la subcategoría con mayor presencia es Reconocer (superior al 50\%) y la con menor presencia es Describir.

Con respecto a las preguntas que demandan un alto nivel cognitivo, en ambas asignaturas se concentran en la habilidad de Analizar, exclusivamente en la subcategoría Explicar; sin embargo, estas preguntas no superan el 20\%. Es importante destacar que las habilidades de Evaluar y Crear, que son superiores a Explicar, no registraron presencia de preguntas. La Tabla 2 presenta información específica sobre la distribución de las preguntas formuladas por la docente y las habilidades cognitivas que estas promueven. 
TABLA 2.

Preguntas del docente y las habilidades cognitivas que promueve

$\begin{array}{llll}\text { Habilidad } & \text { Subcategoría } & \text { Lenguaje \% } & \text { Matemáticas \% } \\ \text { Recuperar } & \text { Reconocer Reproducir Nombrar Describir } & 56,609,61,2 & 64,15,16,40 \\ \text { Comprender } & \text { Exponer Secuenciar Definir Sustituir } & 1,2014,50 & 0000 \\ \text { Aplicar } & \text { Ejecutar Transferir Hacer hipótesis } & 000 & 10,300 \\ \text { Analizar } & \text { Explicar Comparar Categorizar Sintetizar } & 16,9000 & 14,1500 \\ \text { Evaluar } & \text { Juzgar Identificar } & 00 & 00 \\ \text { Crear } & \text { Producir algo nuevo } & 0 & 0\end{array}$

Fuente: Elaboración propia, basada en Tornero et al. (2015)

\section{DiscuSión Y CONCLUSIONES}

Los resultados de esta investigación han permitido develar diversos aspectos que componen el discurso docente. A través del análisis de dos unidades didácticas (una perteneciente a la asignatura de Matemáticas y la otra, a la asignatura de Lenguaje), se buscó identificar el tiempo promedio del habla de la docente, indagar en la intencionalidad de su discurso y analizar las preguntas emitidas según su apertura, y habilidades cognitivas.

Dentro de los principales resultados de esta investigación, se evidencia que el tiempo de habla es dominado principalmente por la docente, siendo este resultado similar a los establecidos por Mercer (2001), Pontefrac y Hardman (2005), Martinic et al. (2013) y Hardman et al. (2012). Con respecto a la intencionalidad del habla, es posible evidenciar que la docente ocupa aproximadamente un tercio de las clases en aspectos relacionados con tareas y procedimientos, y menos de un $10 \%$ en retroalimentar a sus estudiantes. Los resultados explicitados previamente evidencian que predominan actividades dirigidas por la docente, en donde es ella quien lidera una gran parte de los momentos de las clases, relegando a un plano secundario el aprendizaje activo de los propios estudiantes (Martinic et al., 2013, Hardman et al. 2012; Pontefrac y Hardman, 2005). Con respecto a las preguntas emitidas por la docente, en ambas asignaturas es posible evidenciar en primer lugar, que tienen una baja presencia en las clases, correspondiendo solamente al 11,2\% del tiempo total, lo que es prácticamente un 50\% menos a lo establecido por Ajaja y Eravwoke (2012). En segundo lugar, el estudio evidencia que de las preguntas realizadas existe un amplio predominio de aquellas de carácter cerrado y que promueven un bajo nivel cognitivo. Asimismo, son escasas las preguntas de carácter abierto formuladas por la docente, lo que dificultaría el desarrollo de habilidades cognitivas de orden superior, esto se condice con lo establecido por Eliasson, Go\#ran y Sørensen (2017), Hardman et al. (2012) y Chin (2006).

Considerando los resultados de la investigación, se aprecia que la docente presenta un discurso cercano a lo transmisivo más que a lo dialógico, siendo este último fundamental para la promoción de un proceso de mediación (Goldrine y Rojas, 2007), para la negociación de los saberes. Es debido a esto que esta investigación espera ser un aporte que permita comprender la importancia del discurso docente que se lleva a cabo en las aulas. A la vez, se considera relevante que las y los docentes comprendan su importancia y diseñen estrategias comunicativas efectivas que promuevan la comprensión y el desarrollo de habilidades de alto nivel cognitivo, utilizándolas como un vehículo que catalice el aprendizaje, tal como señalan Pontefrac y Hardman (2005). Además, es necesario tener conocimiento de la importancia del rol de las preguntas en el aprendizaje, puesto que son una herramienta psicológica que permite mediar la construcción del conocimiento (Chin, 2006), la comunicación, potenciar habilidades cognitivas, evaluar y monitorear la progresión de las y los estudiantes (Tornero et al., 2015). Lo anterior revela la importancia de que este aspecto sea trabajado desde el proceso de formación inicial de los y las docentes.

Como toda investigación, esta no estuvo exenta de limitaciones. La mayor se relaciona con el hecho de haber realizado el estudio y análisis de una sola docente, lo que hace que los resultados sean específicos y no 
generalizables, entregando una visión muy particular y específica del fenómeno estudiado. Otra limitación fue que las clases fueron audiograbadas y no videograbadas, no permitiendo, por ejemplo, obtener información relacionada con quiénes interactuaba la docente de manera específica.

Los resultados de este estudio revelan nuevas aristas que serían interesantes de investigar a futuro. Frente a esto, en una próxima instancia sería interesante centrarse en el habla de las y los estudiantes, identificando características de su habla, su intencionalidad y las preguntas que realizan en las clases. A la vez, sería interesante indagar en su participación, específicamente en los elementos que influyen en que participe o no participe activamente de las clases. Una tercera proyección sería ampliar esta investigación a un número mayor de docentes que tengan características diversas, con el fin de obtener tendencias de su discurso en nuestro país.

\section{REFERENCIAS}

Ajaja, P. y Eravwoke, U. (2012). Is soliciting important in science? An investigation of science teacher - student questioning interactions. International Education Studies, 5(1), 191 - 199. doi: http://dx.doi.org/10.5539/ies. v5n $1 \mathrm{p} 191$

Barwell, R. (2015). Formal and informal mathematical discourses: Bakhtin and Vygotsky, dialogue and dialectic. Educational Studies in Mathematics, 92(3), 331-345. doi: http://dx.doi.org/10.1007/s10649-015-9641-z

Chen, J. y Liang, X. (2017). Teachers' literal and inferential questions and children's responses: a study of teacherchild linguistic interactions during whole-group instruction in Hong-Kong kindergarten classrooms. [Preguntas literales e inferenciales de los maestros y respuestas de los niños: un estudio de las interacciones lingüísticas entre maestros y niños durante la instrucción de todo el grupo en las aulas de jardín de infantes de Hong Kong]. Early Childhood Education, 45, 671-683. doi: https://doi.org/10.1080/10409289.2018.1556009

Chin, C. (2006). Classroom interaction in science: Teacher questioning and feedback to students' responses. [Interacción en el aula de ciencias: preguntas del profesor y comentarios sobre las respuestas de los alumnos]. International Journal of Science Education, 28(11), 1315-1346. doi: https://doi.org/10.1080/0950069060062 1100

Chin, C. (2007). Teacher questioning in science classrooms: Approaches that stimulate productive thinking. [Interrogatorio docente en las aulas de ciencias: enfoques que estimulan el pensamiento productivo]. Journal of Research in Science Teaching, 44(6), 815-843. doi: https://doi.org/10.1002/tea.20171

Colomina, R., Mayordomo, R. y Onrubia, J. (2001). El análisis de la actividad discursiva en la interacción educativa. Algunas opciones teóricas y metodológicas. Infancia y Aprendizaje, 24, 67-80. doi: https://doi.org/10.1174/02 1037001316899929

Cuadrado, I. y Fernández, I., (2008). ¿Cómo intervienen maestros y profesores para favorecer el aprendizaje en Secundaria? Un estudio comparativo desde el análisis del discurso. Infancia y Aprendizaje, 31, 3-23. doi: https: //doi.org/10.1174/021037008783487101

Díez-Palomar, J., García, P., Molina, S. y Rué Rosell, L. (2010). Aprendizaje dialógico en las matemáticas y en las ciencias. Revista Interuniversitaria de Formación del Profesorado, 24(1), 75-88. Recuperado de https://bit.ly/2 PCjxdp

Eliasson, N., Go\#ran, K. y Sørensen, H. (2017). The role of questions in the science classroom - how girls and boys respond to teachers' questions. [El papel de las preguntas en el aula de ciencias: cómo responden las niñas y los niños a las preguntas de los maestros]. International Journal of Science Education, 39(4), 433-452. doi: https:// doi.org/10.1080/09500693.2017.1289420

Evnitskaya, N. y Berger, E. (2017). Learners' multimodal display of willingness to participate in classroom interaction in the L2 and CLIL context. [Exhibición multimodal de los alumnos de disposición a participar en la interacción en el aula en el contexto L2 y CLIL]. Classroom Discourse, 8(1), 71-94. doi: https://doi.org/10.1080/1946301 4.2016.1272062 
Fleiss, J. (1981). Statistical methods for rates and proportions. [Métodos estadísticos para tasas y proporciones]. New York: John Wiley and Sons.

Godoy, M. (2015). Las preguntas de docentes como estrategia para el desarrollo de habilidades cognitivas de los estudiantes en la asignatura Historia, Geografía y Ciencias Sociales. Foro Educacional, 24, 57-76. Recuperdo de: https://dialnet.unirioja.es/servlet/articulo?codigo $=6429420$

Godoy, F., Varas, L., Martínez, M., Treviño, E. y Meyer, A. (2016). Interacciones pedagógicas y percepción de los estudiantes en escuelas chilenas que mejoran: una aproximación exploratoria. Estudios pedagógicos, 42(3), 149-169. doi: https://dx.doi.org/10.4067/S0718-07052016000400008

Goldrine, T. y Rojas, S. (2007). Descripción de la práctica docente a través de la interactividad profesor-alumnos. Estudios pedagógicos, 33(2), 177-197. doi: https://dx.doi.org/10.4067/S0718-07052007000200010

Hardman, F., Abd-Kadir, J. y Tibuhinda, A. (2012). Reforming teacher education in Tanzania. [Reforma de la formación del profesorado en Tanzania]. International Journalof Educational Development, 32, 826-834. doi: https://doi.org/10.1016/j.ijedudev.2012.01.002

Hus, V. y Kordigel, M. (2011). Questioning as a mediation tool for cognitive development in early science teaching. [El cuestionamiento como herramienta de mediación para el desarrollo cognitivo en la enseñanza temprana de las ciencias].Journal of Baltic Science Education, 10(1), 6-16. Recuperado de https://bit.ly/2PzW6kZ

Lee, Kinzie y Whittaker (2012). Impact of online support for teachers' open-ended questioning in pre-k science activities. [Impacto del apoyo en línea para las preguntas abiertas de los docentes en actividades de ciencias de prekínder]. Teaching and Teacher Education, 28, 568-577. doi: https://doi.org/10.1016/j.tate.2012.01.002

Martinic, S. (2015). El tiempo y el aprendizaje escolar la experiencia de la extensión de la jornada escolar en Chile. Revista Brasileira de Educação, 20(61), 479-499. doi: https://doi.org/10.1590/S1413-24782015206110

Martinic, S. y Vergara, C. (2007). Gestión del tiempo e interacción del profesor-alumno en la sala de clases de establecimientos con jornada escolar completa en chile. Reice. Revista Iberoamericana sobre Calidad, Eficacia y Cambio en Educación, 5(5), 3-20. Recuperado de https://dialnet.unirioja.es/servlet/articulo?codigo=2514189

Martinic, S., Vergara, C. y Huepe, D. (2013). Uso del tiempo e interacciones en la sala de clases. Un estudio de casos en Chile. Pro-Posic\#o\#es, 24(70), 123-135. Recuperado de https://bit.ly/3rlTy7Z

Mercer, N. (2001). Words and minds. [Palabras y mentes]. Londres: Routledge

Mourshed, M., Chijioke, C. y Barber, M. (2012). Cómo continúan mejorando los sistemas educativos de mayor progreso en el mundo. PREAL, Serie de Documentos (61), 1-123. Recuperado de http://200.6.99.248/ bru4 87cl/files/McK61.pdf

Muhonen, H., Rasku-Puttonen, H., Pakarinen, E., Poikkeus, A. y Lerkkanen, M. (2017). Knowledge-building patterns in educational dialogue. [Patrones de construcción del conocimiento en el diálogo educativo]. International Journal of Educational Research, 81, 25-37. doi: https://doi.org/10.1016/j.ijer.2016.10.005

Mumbembe, L., Adewale, A., Peter, I., Adeniyi, M. y Olufunmilayo, O. (2017). An Analysis of a Classroom Discourse: A Case of a Selected English Class in the Eastern Cape of South Africa. [Un análisis de un discurso en el aula: un caso de una clase de inglés seleccionada en el Cabo Oriental de Sudáfrica]. Int J Edu Sci, 12(2), 113-119. doi: https://doi.org/10.1080/09751122.2016.11890418

Oliveira, A. (2010). Improving Teacher Questioning in Science Inquiry Discussions Through Professional Development. [Mejoramiento de las preguntas de los docentes en las discusiones de investigación científica a través del desarrollo profesional]. Journal of Research In Science Teaching, 47(4), 422-453. doi: https://doi.org $/ 10.1002 /$ tea.20345

Pontefract, C. y Hardman, F. (2005). The Discourse of Classroom Interaction in Kenyan Primary Schools. [El discurso de la interacción en el aula en las escuelas primarias de Kenia]. Comparative Education, 41(1), 87-106. doi: htt ps://doi.org/10.1080/03050060500073264

Poulou, M. (2015). Teacher-Student Relationships, Social and Emotional Skills, and Emotional and Behavioural Difficulties. [Relaciones profesor-alumno, habilidades sociales y emocionales, y dificultades emocionales y de comportamiento]. International Journal of Educational Psychology, 4(1), 84-108. doi: http://dx.doi.org/10.44 $71 /$ ijep.2015.04 
Preiss, D., Larraín, A. y Valenzuela, S. (2011). Discurso y Pensamiento en el Aula Matemática Chilena. Psykhe, 20(2), 131-146. doi: https://doi.org/10.4067/S0718-22282011000200011

Prieto, M. (2005). La participación de los estudiantes: ¿Un camino hacia su emancipación?. Theoria, 14(1), 26-36. Recuperado de https://www.redalyc.org/pdf/299/29900104.pdf

Pontefrac, C. y Hardman, F. (2005). The discourse of classroom interaction in Kenyan primary schools. [El discurso de la interacción en el aula en las escuelas primarias de Kenia]. ComparativeEducation.41(1), 87-106. doi: http s://doi.org/10.1080/03050060500073264

Radovic, D. y Preiss, D. (2010). Patrones de Discurso Observados en el Aula de Matemática de Segundo Ciclo Básico en Chile. Psykhe, 19(2), 65-79. Recuperado de https://scielo.conicyt.cl/pdf/psykhe/v19n2/art07.pdf

Reinsvold, L. y Cochran, K. (2011). Power Dynamics and Questioning in Elementary Science Classrooms. [Dinámica de poder y preguntas en las aulas de ciencias elementales]. Journal of Science Teacher Education, 23, 1-24. doi: h ttps://doi.org/10.1007/s10972-011-9235-2

Rey-Herrera, J. y Candela, A. (2013). La construcción discursiva del conocimiento cienti\#fico en el aula. Educación y Educadores, 16(1), 41-65. Recuperado de https://www.redalyc.org/pdf/834/83428614002.pdf

Rusk, F., Sahlström, F. y Pörn, M. (2017). Initiating and carrying out L2 instruction by asking known-answer questions: Incongruent interrogative practices in biand multilingual peer interaction. [Iniciando y llevando a cabo la instrucción L2 haciendo preguntas de respuesta conocida: prácticas interrogativas incongruentes en la interacción biand y multilingüe entre pares]. Linguistics and Education, 38, 55-67. doi: https://doi.org/10.10 16/j.linged.2017.02.004

Sandoval, C. (1996). Investigación cualitativa. En Programa de Especialización en Teorias, Métodos y Técnicas de Investigación Social. Colombia: Instituto colombiano para el fomento de la Educación.

Scott, W. (1998). Organizations. [Organizaciones]. Rational, Natural and Open Systems (4th ed.). New Jersey: Prentice-Hall, Inc.

Sedova, M., Sedlacek, M. y Svaricek, R. (2016). Teacher Professional Development as a Means of Transforming Student Classroom Talk. [Desarrollo profesional docente como medio para transformar la charla en el aula del alumno]. Teaching and Teacher Education, 57, 14-25. doi: https://doi.org/10.1016/j.tate.2016.03.005

Smart, J. y Marshall, J. (2013). Interactions between classroom discourse, teacher questioning and student cognitive engagement in middle school science. [Interacciones entre el discurso en el aula, las preguntas de los maestros y el compromiso cognitivo de los estudiantes en la ciencia de la escuela intermedia]. Journal of Science Teacher Education, 24, 249-267. doi: https://doi.org/10.1007/s10972-012-9297-9

Tornero B., Ramaciotti A., Truffello A., Valenzuela F. (2015). Nivel cognitivo de las preguntas que formulan las educadoras de pa\#trulos. Educación y Educadores, 18(2), 261-283. doi: https://doi.org/10.5294/edu.2015.18. 2.5

Torres, J. y Perera, V. (2009). Cálculo de la fiabilidad y concordancia entre codificadores de un sistema de categorías para el estudio del foro online en e-learning. Revista de Investigación Educativa, 27(1), 89-103. Recuperado de http://revistas.um.es/rie/article/view/94291

Villalta, M. y Martinic, S. (2011). Una aproximación desde la práctica y discurso del docente. Universitas Psychologica, 12(1), 221-233. Recuperado en http://www.scielo.org.co/pdf/rups/v12n1/v12n1a20.pdf

Villalta, M., Martinic, S., Assael, C., Aldunate, N (2018). Presentación de un modelo de análisis de la conversación y experiencias de aprendizaje mediado en la interacción de sala de clase. Revista Educación, 42(1), 2018. Recuperado de https://bit.ly/2O6elxT

Villalta, M., Martinic, S., Guzma\#n, M. (2011). Elementos de la interaccio\#n dida\#ctica en la sala de clase que contribuyen al aprendizaje en contexto social vulnerable. Revista Mexicana de Investigacio\#n Educativa, 16(51), 1137- 1158. Recuperado de https://bit.ly/2O3JcuX

Villalta, M. y Palacios, D. (2014). Discurso y práctica pedagógica en contextos de alto rendimiento escolar. Estudios pedagógicos, 40(2), 373-389. doi: https://doi.org/10.4067/S0718-07052014000300021 
Wells, G. y Meji\#a-Arauz, R. (2005). Toward dialogue in the classroom: Learning and teaching through Inquiry. [Hacia el diálogo en el aula: aprendizaje y enseñanza a través de la investigación]. Working Papers on Culture, Education and Human Development, 1(4), 1-45. Recuperado de https://bit.ly/3kL5EFe

Willemsen, A., Gosen, M., van Braak, M., Koole, T. y de Glooper, K. (2018). Teachers' open invitations in whole-class discussions. [Invitaciones abiertas de maestros en debates de toda la clase]. Linguistics y Education, 45, 40-49. Recuperado de https://core.ac.uk/download/pdf/153218237.pdf

Zuleta, O. (2005). La pedagogía de la pregunta. Una contribución para el aprendizaje. Educere, 9(28), 115-119. Recuperado de https://www.redalyc.org/pdf/356/35602822.pdf

\section{INFORMACIÓN ADICIONAL}

Cómo citar: Pérez-Sobarzo, F. y Yancovic-Allen, M. (2021). El discurso docente en clases de lenguaje y matemáticas en un tercero básico chileno: un estudio de caso. Revista Educación, 45(2). Recuperado de htt p://doi.org/10.15517/revedu.v45i1.42376 